\title{
Sources et moyens de réduction du stress chez les étudiants en médecine : analyse d'entretiens focalisés
}

\section{Sources and ways of reducing stress among medical students: a focus group analysis}

\author{
Flore MORNEAU-SÉVIGNY ${ }^{1}$, Sylvie DODIN ${ }^{2,3}$, Guillaume LAMONTAGNE ${ }^{1}$, \\ Lucie ROCHEFORT $^{2}$ et Geneviève BELLEVILLE ${ }^{1}$ \\ 1 École de psychologie, Université Laval, Québec, Canada \\ 2 Faculté de médecine, Université Laval, Québec, Canada \\ 3 Centre de recherche Hôpital St-François d'Assise, Québec, Canada
}

Manuscrit reçu le 5 juillet 2012 ; commentaires éditoriaux formulés aux auteurs le 14 octobre 2012 ; accepté pour publication le 30 octobre 2012

\author{
Mots-clés \\ Stress ; anxiété : \\ étudiants en \\ médecine ; groupe de \\ discussion focalisée
}

Keywords

Stress; anxiety; medical students; focus groups
Résumé - Contexte : Le stress et l'anxiété représentent une problématique importante chez les étudiants en médecine. À la fin de leur formation, $48 \%$ des étudiants rapportent être anxieux ou nerveux. Objectif : Cette étude explore les besoins, les attitudes et les perceptions des étudiants quant au phénomène du stress et de l'anxiété, afin de mettre en place un programme d'intervention à la faculté de médecine de l'Université Laval. Méthode : Lors de deux entretiens focalisés, treize étudiants du cursus préclinique ont été interrogés à partir d'une entrevue semistructurée qui explorait les thèmes suivants : les sources de stress, la gestion du stress et les choix d'intervention. Résultats : L'anticipation de l'externat et de la résidence, la comparaison entre les étudiants, la performance et les commentaires de certains professeurs sont les principales sources de stress rapportées. Plus spécifiquement, les étudiants craignent de ne pas être en mesure d'appliquer de façon appropriée leurs apprentissages à l'externat et à la résidence. La comparaison des étudiants entre eux porte sur l'avancement dans les études et l'implication extrascolaire. Les étudiants identifient les proches, le sport, la planification du temps et le fait d'avoir effectué une première année dans un autre programme comme des facteurs diminuant le stress. Selon certains, un programme de gestion du stress obligatoire préviendrait le stress. Conclusion : Cette étude révèle que les étudiants en médecine du Québec sont confrontés à plusieurs sources de stress, ce qui justifie la mise en place d'un programme de gestion du stress et de l'anxiété.

Abstract - Context: Stress and anxiety are major issues for medical students. In fact, stress levels increase during medical training. At the end of their training, $48 \%$ of students report feeling anxious or nervous. Objective: This study explores the needs, attitudes and perceptions of students as they relate to stress and anxiety in view of implementing an 
intervention program at the medical school of Laval University. Method: Two focus groups comprising thirteen preclinical students underwent semi-structured interviews focusing on sources of stress, stress management and intervention options. Results: Anticipating residency and clerkships, comparisons between students, performance and some teachers' comments were the main sources of stress reported. Students were specifically worried about not being able to appropriately apply what they learned during residency and clerkships. Comparisons between students focused on progress through the program and extra-curricular involvement. Students identified their family, sports, time management and a first year in another program as stress-reducing factors. According to some students, a mandatory program would help them manage and prevent stress. Conclusion: This study supports the contention that medical students in Laval University's school of medicine face many sources of stress that justify implementing a stress and anxiety management program.

\section{Introduction}

Le stress est un état émotionnel engendré par des causes extérieures et objectives sur lesquelles il est possible d'agir. Dans le cadre de cet article, le terme stress fait référence à un état émotionnel déclenché par des causes externes épisodiques ou chroniques. L'anxiété correspond à un état émotionnel négatif démesuré par rapport aux causes extérieures l'ayant engendrée. Dans ce sens, l'anxiété possède un caractère plus pathologique que le stress ${ }^{[1]}$.

Le stress et l'anxiété consituent une problématique importante chez les étudiants en médecine ${ }^{[2]}$. Le niveau de difficulté associé au programme de médecine favorise une augmentation de l'anxiété ${ }^{[3]}$. Le stress découlerait de plusieurs facteurs tels que le surplus de travail, la compétition et l'exposition à la souffrance humaine ${ }^{[2]}$. Les étudiants de première année rapportent que leur formation en médecine leur crée davantage de stress que leurs problèmes personnels ${ }^{[4]}$. Le stress élevé de ces étudiants peut entraîner une augmentation de l'anxiété, des troubles du sommeil et une diminution du bien-être psychologique. De plus, le niveau d'anxiété augmente au cours de la formation médicale. En effet, à la fin de leur formation, $48 \%$ des étudiants rapportent être anxieux ou nerveux, alors qu'en première année d'étude cette prévalence est de $4 \%{ }^{[5]}$. La détresse psychologique des étudiants en médecine peut entraîner plusieurs conséquences graves comme une diminution de la performance académique, une augmentation de l'abus de substances psychotropes et un déclin de la pratique empathique et du professionnalisme $\mathrm{e}^{[2,6]}$.
Certains auteurs soulignent la nécessité de mettre en place des programmes de gestion du stress dans le cadre de la formation médicale ${ }^{[5]}$. Récemment, des thérapies psychologiques préventives réalisées aux États-Unis et en Norvège ont montré leur efficacité pour réduire l'anxiété et le stress chez les étudiants en médecine. Ces approches consistaient, entre autres, en des groupes de discussion et des techniques de relaxation ${ }^{[7,8]}$. À notre connaissance, au Québec, aucun programme structuré et évalué à l'aide d'outils standardisés n'a été développé dans le but de réduire le stress et l'anxiété chez ces étudiants.

L'objectif de cette étude est d'explorer les besoins, les attitudes et les perceptions des étudiants quant aux phénomènes du stress et de l'anxiété, afin de mettre en place un programme d'intervention à la faculté de médecine de l'Université Laval.

\section{Matériel et méthode}

Des entretiens de groupes focalisés (focus groups) ont été réalisés. L'entretien focalisé comprend un ensemble de discussions réalisées dans un environnement convivial et permissif. Il vise à déterminer les opinions et les intérêts d'une population sélectionnée ${ }^{[9]}$. Deux entretiens focalisés ont été réalisés à l'Université Laval auprès d'étudiants à temps plein du cursus préclinique $\left(1^{\mathrm{re}}, 2^{\mathrm{e}}\right.$ et $3^{\mathrm{e}}$ années). Au total, treize étudiants québécois en médecine de l'Université Laval, soit quatre hommes et neuf femmes, dont l'âge moyen était de 21,5 ans, ont participé à ces entretiens. Dix autres étudiants 
avaient manifesté leur intérêt mais se sont désistés pour cause de conflit d'horaire. Près de la moitié des étudiants interrogés avaient suivi une année d'études dans un autre programme avant de commencer leur doctorat en médecine $(n=6)$. Près du tiers des étudiants interrogés travaillaient durant la session $(n=4)$. Les étudiants ont été recrutés par une annonce réalisée en classe et par courriel.

Les entretiens focalisés portaient sur trois thèmes : les sources de stress, la gestion du stress et les choix d'intervention. Chaque entretien était d'une durée d'une heure trente. Ces entretiens avaient pour but d'établir les sources et les moyens de gestion du stress des étudiants du cursus préclinique, ainsi que leurs expériences relatives à ces thèmes. Concernant le thème du choix d'intervention, les étudiants étaient invités à discuter de diverses techniques de gestion du stress tels que la relaxation ou la gestion des pensées. Ils avaient également la possibilité de se prononcer sur les modalités d'intervention qui conviendraient à leurs besoins. Les participants pouvaient aussi déterminer s'ils préféraient un programme d'intervention facultatif ou obligatoire.

Les entrevues de groupe, de type semi-structuré, ont été conduites à partir d'un guide d'entretien formulé sous la forme de dix questions divisées en trois thèmes. Ces questions ont été élaborées à partir d'une revue de la littérature et des besoins du projet de recherche. Les entretiens focalisés ont été animés par une étudiante en psychologie qui connaît la problématique du stress chez les étudiants en médecine. L'ensemble des étudiants n'a pas répondu aux dix questions ; par contre tous les étudiants se sont prononcés sur chacun des trois thèmes. Les entretiens ont été enregistrés sur fichier audio puis transcrits mot à mot sous forme de verbatim. L'analyse du contenu a été réalisée séparément pour chaque verbatim. Le premier verbatim a été analysé avant de réaliser le second. Chaque verbatim a été décomposé en unités de sens composées de concepts, codifié et analysé par deux étudiants en psychologie à l'aide du logiciel Nvivo. Lorsqu'il y avait discordance de catégorisation, les deux codeurs discutaient de leurs cotations et ce, jusqu'à l'obtention d'un consensus.

La démarche du présent article est inspirée de la méthode phénoménologique car le but était de comprendre le phénomène du stress et de l'anxiété à partir de l'expérience et des perceptions des étudiants, afin d'instaurer un programme d'intervention ${ }^{[10]}$. Les catégories utilisées pour la codification ont été déterminées à la suite d'une première lecture. Les citations de cet article sont celles qui, selon les deux codeurs, illustrent le mieux les idées présentées et qui résument bien le propos ${ }^{[11]}$. Le résumé des principaux résultats a été envoyé à la moitié des participants pour s'assurer de l'intégrité des conclusions tirées.

\section{Résultats}

Les entretiens focalisés ont engendré plusieurs données. Les éléments les plus fréquemment mentionnés sont rapportés dans cette section. Une liste plus exhaustive des catégories de l'analyse de contenu est présentée dans le tableau I. Après l'analyse du premier verbatim, 15 catégories ont été identifiées. À la suite de l'analyse du second verbatim, deux nouvelles catégories ont été ajoutées.

\section{Sources de stress}

L'anticipation par rapport aux années d'externat et de résidence est la principale source de stress chez les étudiants du cursus préclinique. L'externat, d'une durée de 20 mois, comprend notamment des stages en milieu hospitalier. La résidence est d'une durée variable, elle se déroule en milieu hospitalier et permet aux étudiants de se spécialiser. L'idée de mettre en pratique les acquis théoriques du cursus préclinique lors de l'externat et de la résidence engendre du stress. Plusieurs soulignent être inquiets à l'idée d'oublier certains éléments théoriques car la quantité de matière à mémoriser est considérable. Relativement à ce sujet, le participant 3 mentionne : «Le cours que j'ai fait il y a un an, est-ce que je vais m'en rappeler à l'externat? Je commence un peu à avoir un stress préparatoire ». Les responsabilités associées à la pratique médicale lors de l'externat et de la résidence générèrent également un stress chez plusieurs. Le participant 3 avance : « Je pense que c'est un stress de responsabilités [...] de savoir, justement, que dans quelques années on va tomber avec de vrais patients ». 
Tableau I. Catégories de l'analyse de contenu identifies à partir de l'analyse des verbatims des groupes de discussion focalisée.

\begin{tabular}{|c|c|c|}
\hline \multicolumn{2}{|l|}{ Sources de stress } & Facteurs diminuant le stress \\
\hline \multicolumn{2}{|c|}{$\begin{array}{l}\text { - Anticipation de l'externat et de la résidence } \\
\text { - Comparaison et performance } \\
\text { - Commentaires de certains professeurs } \\
\text { - Manque d'information sur les pré-requis associés aux diverses spécialités } \\
\text { - Gestion des horaires associés à la profession } \\
\text { - Image associée à la profession } \\
\text { - Remise en question liée aux éléments stressants de la profession } \\
\text { - Première session et session d'été }\end{array}$} & $\begin{array}{l}\text { - Planification du temps } \\
\text { - Communication avec les proches } \\
\text { - Faire du sport } \\
\text { - Avoir fait une } 1^{\text {re }} \text { année dans un autre } \\
\text { programme } \\
\text { - Être capable d'équilibrer loisir étude }\end{array}$ \\
\hline Interventions les plus appréciées & Modalités d'intervention & $\begin{array}{l}\text { Programme obligatoire versus } \\
\text { optionnel }\end{array}$ \\
\hline $\begin{array}{l}\text { - Techniques de gestion des pensées } \\
\text { et techniques de relaxation }\end{array}$ & $\begin{array}{l}\text { - Certains apprécieraient que le pro- } \\
\text { gramme soit disponible sur Internet. } \\
\text { D'autres que les interventions soient } \\
\text { réalisées par des gens du milieu } \\
\text { médical }\end{array}$ & $\begin{array}{l}\text { - Programme obligatoire : prévient le } \\
\text { stress et rejoint les gens qui ne vont pas } \\
\text { chercher de l'aide } \\
\text { - Programme optionnel : une minorité } \\
\text { d'étudiants sont stressés }\end{array}$ \\
\hline
\end{tabular}

Dans le même ordre d'idées, plusieurs croient ne pas être suffisamment informés des pré-requis nécessaires pour accéder à l'externat et aux programmes de résidence désirés. Plusieurs s'interrogent relativement aux notes et à l'expérience clinique requise pour atteindre leurs objectifs professionnels. L'étudiant 10 soutient : « Moi j'aimerais ça que l'on mette cartes sur table [...]. Si vous visez telle spécialité, enlignez votre préclinique sur telle option. Le bénévolat ils en parlent un peu, mais à quel point ça va être utile à l'externat? »

La comparaison entre les étudiants et les normes associées à la performance engendrent également du stress et de l'anxiété. Concernant la comparaison, les étudiants mentionnent se comparer entre eux davantage sur l'avancement de leur étude et sur l'implication extrascolaire que sur les notes obtenues aux examens. Le participant 11 souligne : «Ce n'est pas tant les notes [qui engendrent le stress], mais c'est plus l'avancement dans l'étude». L'étudiant 9 abonde dans ce sens : "Moi la différence entre un A puis un $\mathrm{A}+$ ce n'est pas ça qui me stresse. C'est plus lorsque je me compare à la personne qui a fait un stage international et qui a plus d'expériences ». Les étudiants trouvent également difficile de se retrouver parmi des individus aussi performants qu'eux. Le participant 6 soulève ce point : "C'est stressant de se retrouver avec du monde autant sur le même pied d'égalité ».

Les commentaires de certains professeurs entrầnent également du stress chez les étudiants. Ces commentaires portent, entre autres, sur les difficultés associées à la profession de médecin. Relativement à ce sujet, le participant 7 avance que : «Parfois il y a aussi des profs qui nous disent, en médecine [il] faut que tu comprennes qu'il faut sacrifier un peu ta vie personnelle ». Ce type de propos engendre une vision négative et anxiogène de la profession.

Certains se demandent s'ils pourront intégrer leurs caractéristiques personnelles à l'image traditionnelle du médecin. Lorsqu'ils considèrent les responsabilités associées à la profession, plusieurs étudiants se demandent s'ils désirent réellement devenir médecins. Ces interrogations engendrent chez eux un stress. Le participant 8 soulève ce point : «J'ai peur de ne pas être capable de respecter mes valeurs familiales et tout ça, en voulant être un bon médecin. Ça me stresse. C'est à partir de ça que je me remets en question ». L'horaire de travail semble également générer du stress et amener les étudiants à se questionner quant à la spécialisation qu'ils désirent effectuer. L'étudiant 13 s'interroge : «Est-ce que je serais mieux de finir pathologiste et de faire du $8 \mathrm{~h}$ à 
$5 \mathrm{~h}$, alors que j'ai toujours voulu être chirurgien orthopédique? ».

\section{Gestion du stress}

Pour gérer leur stress, les étudiants discutent avec leurs proches, font du sport et planifient leur horaire. En ce qui a trait au sport, le participant 4 avance : «L'exercice physique, c'est ma solution pour gérer l'anxiété de soir ». Concernant la planification du temps, le participant 11 indique : "Moi ça m'aide vraiment de faire des listes, de remplir mon horaire ». Dans le même ordre d'idées, les étudiants ayant effectué une première année à l'université avant d'entreprendre le programme de médecine mentionnent que cette expérience leur a permis de développer des outils pour vaincre le stress. L'étudiant 6 souligne : «Pour mon année en psychologie, ce que j'ai aimé, c'est que ça m’a appris à voir venir le stress ».

\section{Pistes d'intervention}

Les entretiens focalisés ont aidé les chercheurs de la présente étude à cibler les interventions à instaurer dans le cadre d'un futur programme de gestion du stress et de l'anxiété. Plusieurs étudiants aimeraient que des exercices de relaxation et de gestion des pensées leur soient enseignés. Le participant 7 avance : «Moi je trouve que, une description de l'anxiété, des techniques de relaxation, c'est de bonnes idées ».

En ce qui a trait aux modalités d'intervention, l'opinion est divisée. Certains participants aimeraient que le programme soit offert en classe par divers professionnels, tels que des médecins ou des psychologues. D'autres souhaitent que le programme soit accessible par Internet. Concernant la possibilité de mettre en place un programme de gestion du stress, qui serait obligatoire pour tous les étudiants en médecine, les avis sont partagés. Certains croient que seule une minorité d'étudiants est stressée. Par conséquent, un programme obligatoire ne serait pas pertinent. D'autres pensent qu'un programme obligatoire permettrait de prévenir le stress et de soutenir les étudiants qui n'iraient pas chercher de l'aide par eux-mêmes.

\section{Discussion}

Cette étude avait pour but d'explorer les besoins, les attitudes et les perceptions des étudiants en matière de stress et d'anxiété pour mettre en place un programme d'intervention. Cette recherche qualitative a permis de déterminer les sources de stress des étudiants en médecine, leurs techniques de gestion du stress et leurs besoins en matière d'intervention. En effet, la présente étude révèle que l'application pratique des acquis théoriques dans le cadre de l'externat et de la résidence génère du stress chez les étudiants. De plus, les étudiants soutiennent être stressés quant au fait de se retrouver parmi des individus aussi performants qu'eux. Ceux-ci soulignent se comparer entre eux davantage sur l'implication extrascolaire que sur la performance académique. Les commentaires de certains professeurs sur les difficultés liées à la profession engendrent également du stress chez les étudiants. Les étudiants se disent inquiets quant aux horaires et aux responsabilités liés à la pratique de la médecine. Afin de diminuer leur stress, les étudiants planifient leur horaire, font du sport et discutent avec leurs proches.

Cette étude a permis de mettre en lumière l'expérience subjective des étudiants quant au stress et à l'anxiété. Comparativement à une étude quantitative, cette recherche qualitative a permis de dégager de façon nuancée l'ensemble des attitudes, des expériences et des perceptions des étudiants interrogés. Au Québec, peu d'études sur le stress des étudiants en médecine ont été réalisées ; cette recherche comble donc une lacune dans la littérature.

Les résultats de cette recherche sont similaires à ceux obtenus par la Fédération médicale étudiante du Québec. En effet, selon un sondage auquel ont répondu $32 \%$ des externes, les objectifs peu précis et le manque d'information sur les stages sont des sources de stress importantes. Selon ce sondage, la pression de performance, le choix de carrière et le sentiment de culpabilité par rapport aux études font 
partie des cinq plus grandes sources de stress non académiques ${ }^{[12]}$. Ces sources de stress ont été mentionnées lors des entretiens focalisés. Les résultats de notre étude enrichissent la littérature car ils apportent des mises en contexte et des exemples concrets découlant de l'expérience des étudiants en médecine.

Cette étude comporte cependant quelques limites, liées notamment à l'absence de triangulation dans la collecte des données. Un seul type de méthode a été utilisé, en l'espèce le groupe de discussion focalisée. De plus, concernant le nombre d'entretiens focalisés, il est difficile de déterminer si une saturation des données a été obtenue. Par contre, la redondance de catégories soulevées laisse croire que celle-ci a été atteinte. En outre, la rétroaction positive obtenue à la suite de l'envoi d'un résumé des résultats à la moitié des participants accrédite la crédilité des conclusions tirées. La généralisation des résultats est la principale limite de cette étude. Les étudiants interrogés étaient majoritairement des individus recrutés sur la base du volontariat, impliqués au sein du programme de médecine et à l'aise quant à l'idée de discuter du stress et de l'anxiété. Ces individus ne représentent peut-être pas l'ensemble de la population étudiante. Le petit échantillon composé uniquement d'étudiants franco-québécois du cursus préclinique à l'Université Laval et la collecte qualitative des données limitent la possibilité d'extrapoler les conclusions tirées. Cependant, considérant que cette étude est une étape préliminaire à la mise en place d'un programme de gestion du stress à l'Université Laval, des résultats spécifiques à la population visée sont très pertinents.

Les résultats obtenus serviront de base pour l'élaboration d'un programme de gestion du stress et de l'anxiété pour les étudiants de l'Université Laval. Ce programme devra cibler les principales sources de stress mentionnées, telles que l'externat et la résidence. Il devra également prendre en compte les techniques déjà utilisées par les étudiants pour gérer leur stress, comme la planification du temps. Les techniques qui seront mises en place sont celles qui ont obtenu le plus d'appui de la part des élèves, telles que les techniques de gestion des pensées. Pour conclure, cette étude révèle que les étudiants en médecine du Québec sont confrontés à plusieurs sources de stress, ce qui justifie la mise en place d'un programme de gestion du stress et de l'anxiété.

\section{Contributions}

Flore Morneau-Sévigny a rédigé le manuscrit, a recueilli les données et a participé à l'interprétation des résultats. Geneviève Belleville et Sylvie Dodin ont participé à la conception du protocole et à la révision du manuscrit. Guillaume Lamontagne a participé à l'interprétation des résultats. Lucie Rochefort a assumé la liaison entre les chercheurs et la population cible.

\section{Approbation éthique}

Ce projet de recherche a obtenu l'approbation du Comité d'éthique de la recherche avec des êtres humains de l'Université Laval (No. d'approbation 2011-120 A2/2709-2011).

\section{Déclaration d'intérêts}

Aucun auteur ne déclare de conflit d'intérêts relatifs à l'objet de cet article.

\section{Références}

1. Boudarene M. Stress et anxiété : de quoi s'agit-il ? Encéphale 1998;24:557-68.

2. Dyrbye LN, Thomas MR, Shanafelt TD. Systematic review of depression, anxiety, and other indicators of psychological distress among US and Canadian medical students. Acad Med 2005; 1:354-73.

3. Smith CK, Peterson DF, Degenhardt BF, Johnson JC. Depression, anxiety, and perceived hassles among entering medical students. Psychol Health Med 2007;12:31-9.

4. Moffat KJ, McConnachie A, Ross S, Morrison JM. First Year Medical Students Stress and Coping in a Problem-Based Learning Medical Curriculum. Med Educ 2004;38:482-91.

5. Nemi PN, Vainioma, PT. Medical students' distress quality, continuity and gender differences during a six-year medical programme. Med Teach 2006;28:136-141.

6. Thomas MR, Dyrbye LN, Huntington JL, Lawson KL, Novotny PJ, Sloan JA, Shanafelt, TD. How do distress and well-being relate to medical student empathy? A multicenter study. J Gen Intern Med 2007; 22:177-83. 
7. Finkelstein C, Brownstein A, Scott C, Lan Y. Anxiety and stress reduction in medical education: an intervention. Med Educ 2007;41:258-64.

8. Holm M, Tyssen R, Stordal K, Haver B. Self-development groups reduce medical school stress: a controlled intervention study. BioMed Central Education 2012;10:1-8.

9. Krueger R, Casey MA. Focus groups: A practical guide for Applied Research. Californie: Sage, 2009.

10. Côté L, Turgeon J. Comment lire de façon critique les articles de recherche qualitative en médecine. Pédagogie Médicale 2002;3:81-90.
11. Bertrand JT, Brown JE, Ward VM. Techniques for Analyzing Focus Group Data. Evaluation Review: Sage, 1992.

12. Fédération médicale étudiante. Rapport bien-être. 2011 [En ligne]. Disponible sur: http:// www.fmeq.ca/rapport-bien-etre.

Correspondance et offprints : Geneviève Belleville; 2325 rue des Bibliothèques, Québec, Canada.

Mailto : Genevieve.Belleville@psy.ulaval.ca. 